\title{
QUELQUES OBSERVATIONS SUR LES EMPLOIS DISCURSIFS DE LA PRÉPOSITION DE DANS L'OPTIQUE DE LA THÉORIE DES PRÉCÉDENTS
}

\author{
VARVARA AMELICHEVA \\ Université d'État Lomonossov de Moscou, Russie
}

\begin{abstract}
Résumé. L'article est consacré aux groupes nominaux (GN) d'intérêt interculturel avec la préposition de et les noms propres. Des contextes bibliques comme l'échelle de Jacob aux plus récents comme les yeux du Chat Potté, tous évoquent des phénomènes de précédent, dans les termes du linguiste éminent russe Yuri Karaulov. Le comportement syntaxique de ces GN est différent de celui des GN prototypiques. Par exemple, dans la question banale des interviews Quelle est votre madeleine de Proust? le GN de ce type est accompagné d'un adjectif possessif, ce qui rend impossible son interprétation prototypique de possession (madeleine de Proust = qui appartient à Proust). La présente étude, basée sur un petit corpus d'exemples tirés de l'autobiographie de Bernard Pivot, permet de faire quelques observations sur ce type de GN et sur leurs propriétés sémantiques.
\end{abstract}

Mots clés : français, préposition, de, noms propres, phénomène de précédent

\section{INTRODUCTION}

Dans son article désormais classique de 1996, Eléments pour une typologie des SN complexes en de en français, la chercheuse suédoise Inge Bartning oppose les emplois prototypiques de la préposition $d e$, c.-à-d. ceux qui sont facilement décodables à partir des traits sémantiques des substantifs qu'elle relie, aux emplois discursifs dont l'interprétation découle du contexte à un niveau supérieur. L'interprétation des emplois prototypiques se produit au niveau de la micro-structure; le syntagme nominal (SN) est interprété suivant le sens des deux entités nominales, surtout celui du N1, le co-texte gauche de la préposition: le voyage de Luc (agent), du thé de Chine (provenance), la voiture de Pierre (possession), un sac de plage (destination). La valeur de la préposition de dépend donc des rôles dénotatifs des deux entités nominales reliées; la relation préexiste au discours et elle est récupérable à partir des unités lexicales en cause.

Au contraire, l'interprétation des emplois discursifs se produit au niveau de la macro-structure: les entités nominales peuvent soit être présentes antérieurement 
dans le texte, soit appartenir à l'univers du discours global ou à des connaissances extra-linguistiques. Dans les deux cas, l'interprétation demande un contexte plus large que le SN lui-même. En voici quelques exemples proposés par I. Bartning:

(1) Debout devant la cheminée était un homme de moyenne taille, à la mine haute et fière, [...] De temps en temps, l'homme de la cheminée levait les yeux de dessus les écritures (Dumas, Les Trois mousquetaires).

(2) C'est cette photographie qui est au plus près de celle qui n’a pas été faite de la jeune fille du bac (Duras, L'Amant).

(3) Et puis à le voir, lui, l'homme de la Mandchourie endormi ou mort. Celui de la main, celui du voyage (Duras, L’Amant).

(4) Marie admirait une magnifique robe dans la vitrine. Une cliente demanda à essayer la robe de Marie. (Bartning, 1996: 30)

L'intervalle textuel nécessaire pour décoder ces SN peut varier considérablement: pour l'exemple (1) il équivaut à trois phrases, pour (2) à trois pages. Dans l'exemple (3) les deux SN évoquent une même situation: lors d'un voyage, l'homme a posé sa main sur le genou de la jeune fille. Dans l'exemple (4) seul le contexte élargi permet de comprendre qu'il s'agit d'un intérêt particulier de Marie pour cette robe, et donc d'opter pour l'interprétation discursive au lieu de l'interprétation prototypique (celle de possession), qui semble pourtant plus naturelle si on considère ce $\mathrm{SN}$ isolément.

I. Bartning range aussi parmi les emplois discursifs ceux dont l'interprétation demande des connaissances extra-linguistiques ou interculturelles. Nous trouvons que ce type d'emplois de la préposition de mérite une étude approfondie et un statut à part:

(5) Les secrets de famille sont comme ça. Ils vous tiennent comme le sparadrap du capitaine Haddock. Le plus agaçant, sans doute, c'est que tout le monde en a, tout le monde en fabrique, tout le monde en souffre, mais que personne n'a encore trouvé le moyen de s'en débarrasser. (Nouvel Observateur, 1582)

Contrairement à (1-4), dans l'exemple (5) le contexte élargi ne contient aucune information permettant d'interpréter le $\mathrm{SN}$ en $d e$, parce que ce sont les connaissances interculturelles franco-belges qui sont mises en jeu: dans l'épisode L'affaire Tournesol de la bande dessinée de Hergé sur Tintin il s'agissait d'un sparadrap dont le capitaine ne pouvait pas se débarasser. Bien que I. Bartning ne fasse pas la dictinction entre ce contexte et les autres emplois discursifs de la préposition $d e$, nous croyons que la notoriété des éléments de la situation a forcément un impact sur son interprétation; cf. les observations de Ekaterina Rakhilina sur le génitif russe: la phrase Segodnya my budem smotret' film Mashi (Ce soir on va voir le/un film de Macha) peut être interprétée de plusieurs manières: le film dans lequel elle a tourné / qu'elle voulait voir / qu'elle a acheté / dont elle a parlé, alors que Segodnya my budem smotret' film Fellini (Ce soir on 
va voir le/un film de Fellini) sera probablement interprété comme un film qu'il a tourné (Rakhilina, 2010: 249). Dans le cadre de cet article, on présentera quelques observations préliminaires faites sur un mini-corpus d'une soixantaine de contextes du type $\mathrm{N}+d e+$ Npropre.

\section{CORPUS DE L'ÉTUDE}

Dans son livre autobiographique Les mots de ma vie, Bernard Pivot, journaliste, écrivain et animateur d'émissions culturelles à la télévision, parle d'un projet d'émission qui n’a jamais été réalisé:

Un jour, j'eus l'idée d'une émission qui se serait intitulée Le petit quelque chose en plus. Beaucoup de gens illustres ou célèbres, de jadis, d'hier et d'aujourd'hui, ont ajouté aux exploits, aux activités, admirables ou détestables, qui leur ont valu ou qui leur valent leur renommée un petit quelque chose. Ce détail, cette particularité, ce "gimmick", ou mieux cette mini-mythologie, est devenu tellement connu du public qu'il les identifie spontanément. Exemples: le tonneau de Diogène, la moustache de Dalí, la main de Napoléon dans son gilet, le chapeau de Mitterrand, la madeleine de Proust, la chemise blanche échancrée de Bernard-Henri Lévy.

Cette singularité est soit une caractéristique physique, soit un vêtement, soit un accessoire, soit un animal, soit toute chose à laquelle leur image est immédiatement liée, leur réputation indéfectiblement associée.

Cela vaut aussi pour des personnages imaginaires auxquels leurs créateurs ont donné un "truc" original qui ne s'oublie pas. Exemple: le nez de Cyrano.

Toutes les femmes et tous les hommes passés à la postérité ou installés dans la considération du moment n'ont pas "un petit quelque chose en plus". Mais beaucoup le possèdent. Mettez au cours d'un repas la conversation là-dessus et vous constaterez que les convives, piqués au jeu, feront assaut de références. Dans le désordre ils citeront probablement:

Le panache blanc d'Henri IV, la cuisse de Jupiter, l'oreille de Van Gogh, la dictée de Mérimée, les bananes de Joséphine Baker, la moustache de Staline, les yeux de Michèle Morgan, l'épée de Damoclès, les pommes de Cézanne, le bandana de John Galliano, la barbe de Victor Hugo, les lunettes de Trotski, le chapeau de Gaston Defferre, le masque de Zorro, la cigarette de Michel Houellebecq, la pomme de Newton, le sourire de la Joconde, la cafetière de Balzac, le chapeau de Marc Veyrat, les yeux d'Elsa (Triolet), les chats de Léautaud, la canne de Charlot, la tête de veau ou la bière Corona de Chirac, le nez de Cléopâtre, la petite robe noire de Piaf, la surdité de Beethoven, la salopette de Coluche, le cigare de Churchill, le keffieh 
d'Arafat, les seins de Sophie Marceau, le manteau de saint Martin, le chien de Michel Drucker, le fauteuil de Molière, l'imperméable d'Humphrey Bogart, l'imperméable et le cigarillo de l'inspecteur Colombo, la moustache d'Hitler, le tournedos Rossini, la voix éraillée de Mauriac, le chapeau de Monsieur Hulot, la pipe de Simenon et de Maigret, les lunettes de Harry Potter, la pomme de Guillaume Tell, la barbe de Raspoutine, la rose de Nehru, l'entrejambe de Sharon Stone (Basic Instinct), la brouette de Pascal, le mégot au coin des lèvres de Prévert, les lunettes d'Elton John, la frange de Louise Brooks, l'écharpe rouge de Pierre Rosenberg, le canotier de Maurice Chevalier, les loups d'Hélène Grimaud, le parapluie de Chamberlain, le crâne rasée de Yul Brynner, la moustache de José Bové, la poitrine de Mae West, la langue tirée d'Einstein, les lunettes noires, le catogan et les gants de Karl Lagerfeld, le crâne rasé de Barthez ("le divin chauve"), le chapeau et la fleur à la boutonnière de Charles Trenet, la baignoire de Marat, la pomme d'Ève et d'Adam, les chats de Colette [...]. (Pivot, 2011: 75)

Tout littéraire qu'il soit, ce texte prête à des réflections purement linguistiques et représente en même temps un petit corpus d'exemples très précieux.

Tout d'abord, il est à noter que les personnages cités sont assez hétérogènes: parmi eux, on trouve 14 hommes politiques (Henri IV, Staline, Trotski, Gaston Defferre, Chirac, Churchill, Arafat, Hitler, Raspoutine, Nehru, Chamberlain, José Bové, Marat), 10 écrivains (Mérimée, Victor Hugo, Michel Houellebecq, Balzac, Léautaud, Molière, Mauriac, Simenon, Prévert, Colette), 9 chanteurs, musiciens ou compositeurs (Joséphine Baker, Piaf, Beethoven, Rossini, Elton John, Maurice Chevalier, Hélène Grimaud, Mae West, Charles Trenet), 9 personnages d'œuvres d'art (Zorro, Joconde, Charlot, Elsa, inspecteur Colombo, monsieur Hulot, Maigret, Harry Potter, Guillaume Tell), 7 comédiens ou comédiennes (Michèle Morgan, Coluche, Sophie Marceau, Humphrey Bogart, Sharon Stone, Louise Brooks, Jul Brynner), 6 personnages mythologiques (Jupiter, Damoclès, saint Martin, Ėve et Adam, Cléopâtre), 2 peintres (Van Gogh, Cézanne), 2 couturiers (John Galliano. Karl Lagerfeld), un chef cuisinier (Marc Veyrat), un journaliste (Michel Drucker), un historien de l'art (Pierre Rosenberg) et un footballeur (Barthez). Seuls $50 \%$ d'entre eux sont d'origine française à proprement parler; pourtant, ce que tous ont en commun, c'est leur notoriété aux yeux du Français lambda doté tant soit peu de culture générale.

Au niveau formel, les contextes sont tous semblables: mis à part le tournedos Rossini (qui reproduit le même modèle que steak Chateaubriand, sauce Colbert, hachis Parmentier), tous représentent des SN du type $\mathrm{N}+$ de + Npropre. Au niveau sémantique, il est facile à remarquer que le mini-corpus s'organise autour de plusieurs axes suivant les types sémantiques des $\mathrm{N} 1$, dont les plus nombreux sont:

- vêtement ou accessoire vestimentaire (20 contextes),

- partie du corps (18 contextes), 
- animal de compagnie (4 contextes),

- produits du tabac (4 contextes), etc.

Pourtant, il est clair que l'interprétation prototypique (réalisée à l'intérieur du SN et se basant sur les rôles dénotatifs des deux entités nominales l'une par rapport à l'autre) ne suffit pas pour définir la nature exacte des relations exprimées par la préposition $d e$.

Par exemple, on trouve dans ce corpus quatre SN avec l'élément N1 pomme:

- les pommes de Cézanne,

- la pomme de Newton,

- la pomme de Guillaume Tell,

- la pomme d'Ève et d'Adam,

qui évoquent quatre situations complètement différentes, et qui sont pourtant bien compréhensibles, transparentes pour n'importe quel francophone maîtrisant quelques notions de base de la culture européenne (et même pas spécifiquement française). Si les pommes de Cézanne sont le sujet privilégié de ses tableaux, la pomme de Newton nous rappelle la découverte de la gravitation, la pomme de Guillaume Tell a été posée sur la tête de son fils en tant que cible, et la pomme d'Ève et d'Adam fait allusion au péché originel. L'importance de cette information interculturelle se révèle si grande que pour aucun de ces cas de figure, l'interprétation prototypique de possession, la plus probable pour un SN isolé avec un nom propre n'ayant pas de connotations, comme la pomme de Paul, ne peut pas être appliquée, sauf, théoriquement, dans quelque contexte plus large qui pourrait la réactualiser (mais même alors cet emploi resterait discursif!).

\section{PROPOSITIONS POUR L'ANALYSE}

Nous croyons que ces emplois discursifs de la préposition de pourraient être analysés dans l'optique de la théorie des précédents dont les bases ont été jetées par le linguiste éminent russe Yuri Karaulov. Dans son livre de 1987 La langue russe et la personnalité langagière il a introduit le terme 'texte de précédent' dont il se servait pour décrire le portrait langagier d'une personne. D’après lui, les textes de précédent sont '(1) significatifs pour telle ou telle personne sur le plan cognitif ou émotionnel, (2) ayant un caractère "surpersonnel", c.-a-d. bien connus de l'entourage de cette personne, y compris ses prédécesseurs et ses contemporains, et enfin (3), cités par cette personne itérativement, à maintes reprises' (Karaulov 1987: 216; trad. par moi-même). Postérieurement, cette théorie a été mise au point par d'autres linguistes russes (y compris D. B. Gudkov, V. V. Krasnykh, I. V. Zakharenko), qui ont étendu la notion de précédent aux phénomènes de caractère et d'échelle différents. Ces phénomènes peuvent être verbaux (tous types de textes) ou non verbaux (œuvres d’art, événements hystoriques, etc.), mais ils appartiennent forcément à la conscience collective d'une communauté langagière. Dans la présente étude, nous nous intéresserons particulièrement à la notion du nom de précédent. D’après Irina Zakharenko et ses collègues 
(Zakharenko, Krasnykh, Gudkov et Bagaeva, 1997), c'est un nom individuel lié à un texte de précédent (Tartuffe, Cyrano, D'Artagnan) ou à une situation de précédent (personnages historiques comme Charlemagne, Louis IX); c'est une sorte de signe complexe qui ne fait pas référence directement à la personne dénotée, mais plutôt à un ensemble de ses propriétés distinctives et de ses détails accessoires (ibid.: 90).

Ces détails, par exemple les vêtements typiques ou les particularités du physique, permettent d'identifier, de reconnaître spontanément la personne en question à quiconque faisant partie d'une communauté langagière ou culturelle donnée. Ainsi, en parlant à un Russe, il suffit de mentionner un képi, plus une automobile blindée (du haut de laquelle le personnage haranguait la foule), plus le grasseyement pour qu'on comprenne tout de suite qu'il s'agit de Lénine.

La théorie des précédents de Karaulov se rapproche en quelque sorte de celle des stéréotypes de J.-Cl. Anscombre qui constate que les stéréotypes 'nous viennent en particulier de notre éducation, à commencer par l'école, qui est un grand fabricateur de stéréotypes, en particulier dans le domaine historique. Un bon exemple en est l'image de Du Guesclin respectivement dans les livres d'histoire français et espagnols' (Anscombre, 2010: 10). P. Cadiot et Y.-M. Visetti, de leur part, en parlant des motifs linguistiques, font remarquer que 'si l'on appuie à une culture moyenne, on peut penser qu'ours sera moins contraint que Don Juan', ce dernier pouvant impliquer un programme narratif conforme au type bien connu (dans l'ordre, et en boucle: séduire en promettant l'amour, coucher, abandonner, puis recommencer, avec une aura toujours plus forte de défi scandaleux)' (Cadiot et Visetti, 2001: 159). Pourtant, si le phénomène a été signalé par des linguistes français, aucun n’a consacré, autant que nous sachions, d'études particulières aux $\mathrm{SN}$ en de comportant des noms propres de précédent. Cela nous permet d'espérer que cette brève présentation de la théorie des précédents pourrait intéresser le lecteur francophone.

Le contexte le nez de Cléopâtre qui fait partie de notre corpus peut servir de preuve à l'hypothèse que dans les $\mathrm{SN}$ analysés il s'agit bien de noms de précédent, plutôt que des traits particuliers de personnages historiques réels. En effet, on ne sait rien de précis sur la forme du nez de la reine d'Égypte, ce contexte faisant allusion à une citation des Pensées de Blaise Pascal: Le nez de Cléopâtre, s'il eût été plus court, toute la face de la terre aurait changé. De même, le SN les yeux d'Elsa, sans aucun doute, ne renvoie pas le destinataire du message aux yeux réels d'Elsa Triolet, mais au poème de Louis Aragon (Tes yeux sont si profonds qu'en me penchant pour boire / J'ai vu tous les soleils y venir se mirer), contrairement au SN les yeux de Michèle Morgan, actrice de cinéma qui avait effectivement d'énormes yeux bleus. On pourrait y ajouter un autre exemple plus récent avec ce même N1: le SN les yeux du Chat Potté met en valeur une expression spéciale, un regard particulièrement attendrissant du petit chat belliqueux, personnage du dessin animé Shrek. Là encore, on peut constater que l'interprétation adéquate du SN 
relève de la connaissance extra-linguistique partagée par toute une communauté langagière plutôt que du sémantisme du N1.

\section{EN GUISE DE CONCLUSION}

Même si la théorie des précédents ne propose pas d'explication sémantique unique pour tous les SN étudiés (d'ailleurs, une telle explication existe-t-elle?), elle justifie très bien la cohérence pragmatique qui est née du rapprochement d'un nom de précédent et de son détail accessoire au sein d'un syntagme en de.

Au niveau de la micro-structure, cette cohérence permet à de tels contextes de fonctionner comme métaphores ou unités phraséologiques, à commencer par toute une série d'expressions bibliques (l'arche de Noé, l'ânesse de Balaam, les amis de Job, l'échelle de Jacob, etc.). Notamment, la pomme d'Adam, qui, à l'origine, voulait dire 'le fruit défendu', aujourd'hui signifie aussi une partie du corps: la proéminence laryngée. La madeleine de Proust ne signifie plus une pâtisserie, mais tout phénomène déclencheur d'une impression de réminiscence. Au niveau syntaxique, cela se traduit par sa capacité d'être accompagné d'un adjectif possessif (comme dans la question banale des interviews Quelle est votre madeleine de Proust ?), ce qui exclut son interprétation prototypique de possession (madeleine de Proust $=$ qui appartient à Proust). De même, on trouve facilement sur Internet des exemples du SN un nez de Cyrano avec l'article indéfini (Il est doté d'un nez de Cyrano / je peux comprendre les rhinoplasties pour raccourcir un nez de Cyrano), alors que l'interprétation prototypique demanderait d'employer l'article défini.

Pour conclure, on pourrait dire qu'il n'y a pas à s'étonner que ce soit justement de la préposition de avec son sémantisme extrêmement souple et riche que la langue française se sert pour exprimer les relations stables, mais difficiles à définir qui existent entre un nom de précédent et son détail accessoire dans ce type d'emplois discursifs.

\section{REFERENCES}

Anscombre, J.-Cl. (2010) À la croisée des chemins: la théorie des stéréotypes. In C. À. Castro, F. M Bango de la Campa, M. L. Donaire (eds.) Liens linguistiques. Études sur la combinatoire et la hiérarchie des composants (pp. 7-23). Bern: Peter Lang.

Bartning, I. (1996) Éléments pour une typologie des SN complexes en de en français. Langue française, 1 (109): 29-43.

Cadiot, P., Visetti, Y.-M. (2001) Pour une théorie des formes sémantiques. Paris: Presses Universitaires de France.

Karaulov, Y. N. (1987) Russkiy yazyk i yazykovaya lichnost [La langue russe et la personnalité langagière]. Moscow: Nauka.

Pivot, B. (2011) Les mots de ma vie. Paris: Albin Michel.

Rakhilina, E. V. (2010) Lingvistika konstruktsiy [La linguistique des conctructions]. Moscow: Azbukovnik.

Zakharenko, I. V., Krasnykh, V. V., Gudkov, D. B., Bagaeva, D. B. (1997) Pretsedentnoe imya i pretsedentnoe vyskazyvanie kak simvoly pretsedentnykh fenomenov [Le nom de 
précédent et l'énoncé de précédent en tant que symboles de phénomènes de précédent]. In V. V. Krasnykh, A. I. Izotov (eds.) Yazyk, soznanie, kommunikatsiya [Langue, Conscience, Communication]. Vol. 1 (pp. 82-103). Moscow: Filologiya.

\title{
SOME OBSERVATIONS ON THE DISCURSIVE USE OF THE PREPOSITION DE UNDER THE FRAME OF THE THEORY OF PRECEDENT PHENOMENA
}

\begin{abstract}
The article deals with French noun groups (NGs) including the preposition de and proper nouns that represent an intercultural interest. From biblical contexts such as l'échelle de Jacob to more recent ones like les yeux $d u$ Chat Potté, all evoke precedent phenomena, in the terms of the eminent Russian linguist Yuri Karaulov. The syntactic behavior of these NGs is also different from that of prototypical NGs. For example, in the banal question of interviews Quelle est votre madeleine de Proust? (What is your Proust's madeleine?) the NG of this type is accompanied by a possessive adjective, which excludes its prototypical interpretation of possession (madeleine de Proust $=$ which belongs to Proust). Our study, based on a small corpus of examples found in the autobiography of Bernard Pivot, permits to make some observations on this type of NGs with de and on their properties.
\end{abstract}

Key words: French, preposition, de, proper nouns, precedent phenomena

Varvara Amelicheva (docteur ès lettres, maître de conférences au département de linguistique française) travaille à l'Université d'Etat Lomonossov de Moscou. Son domaine d'études inclut le sémantisme et les particularités d'emploi des prépositions françaises. Email: varvara.amelicheva@gmail.com 